\title{
Der praktische Nutzen von Registerdaten
}

BioReg, das österreichische Biologika-Register für entzündliche rheumatische Erkrankungen, wurde vor 12 Jahren gegründet. Priv.-Doz. Dr. Burkhard Leeb, Herausgeber der rheuma plus und Mitbegründer des Registers, erklärt im Gespräch: Warum sind - trotz der vielen Studiendaten zu neuen Medikamenten - Registerdaten wichtig? Und was lässt sich zur Therapie rheumatischer Erkrankungen und zur rheumatologischen Versorgung in Österreich aufgrund von Registerdaten sagen.

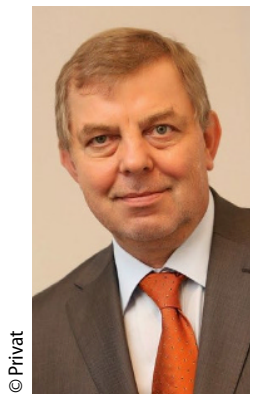

Priv.-Doz. Dr. Burkhard Leeb
Welche Daten werden im BioReg erfasst und zu welchem Zweck?

LEEB: BioReg ist ein Register zur Aufzeichnung der Therapie entzündlich rheumatischer Erkrankungen mit Biologika, Biosimilars und targeted synthetic (ts)DMARDs, früher auch als Small Molecules bekannt. Daneben wird auch die konventionelle Basistherapie, die viele Patienten zur gleichen Zeit erhalten, im BioReg erfasst. Eine etwaige Therapie mit Kortison wird ebenso registriert wie die Gabe von Analgetika und nicht-steroidalen Antirheumatika.

BioReg ist ein landesweiter Zusammenschluss von Rheumatologen und Rheumatologinnen in über ganz Österreich verteilten rheumatologischen Zentren, die Daten zu Patienten in eine zentrale Datenbank eingeben. Der Fokus liegt auf Therapiesicherheit und Effektivität - besser:Therapie-Effizienz, nämlich der Zusammenschau aus Effektivität, Therapieadhärenz und Sicherheit.

Welche Indikationen werden im BioReg erfasst?

LEEB: Erfasst werden die Indikationen rheumatoide Arthritis, Spondylitis ankylosans, Arthritis psoriatica und sonstige entzündliche rheumatische Erkrankungen - das sind alle anderen entzündlichen rheumatischen Erkrankungen, bei denen Biologika, Biosimililars oder tsDMARDs eingesetzt werden. Diese Patientengruppe wird seit etwa sechs Jahren dokumentiert, um festzuhalten bei welchen Erkrankungen jene Medikamente sonst noch zum Einsatz kommen; es finden sich in dieser Kohorte Patienten und Patientinnen mit Polymyalgia rheumatica, Lupus erythematodes, Granulomatose mit Polyangitis etc. Biologika, Biosimilars und tsDMARDs kommen dabei, teils auch Off Label, zum Einsatz.

\section{Wer sammelt die Daten, die in BioReg} aufscheinen?

LEEB: Insgesamt liefern die BioRegMitglieder - Spitalsabteilungen, Ambulatorien, Rehabilitationszentren und Privatordinationen Daten. Pro Institution speisen mitunter mehrere Rheumatologen Daten ins System ein. Die Aktivität der Mitglieder ist zeitlich und auch was die Patienten-Zahlen betrifft, unterschiedlich. Und natürlich wäre es wünschenswert, wenn noch mehr Kolleginnen und Kollegen Daten in BioReg eingeben würden.

\section{Wie wird BioReg finanziert?}

LEEB: BioReg finanziert sich durch einen unrestricted Grant der pharmazeutischen Industrie. $\mathrm{Zu}$ den Unterstützern gehören ABBVIE GmbH, AMGEN GmbH, BIOGEN GmbH, ELI LILLY GmbH, FRESENIUS-KABI AUSTRIA GmbH, JANSSEN-CILAG PHARMA GmbH, MERCK SHARP \& DOHME GmbH, PFIZER CORPORATION AUSTRIA $\mathrm{GmbH}$, ROCHE AUSTRIA GmbH, SANDOZ GmbH und UCB PHARMA $\mathrm{GmbH}$. Die unterstützenden Firmen haben keinerlei Einfluss auf die Datenerhebung und Datenanalyse sowie die Auswahl der Patienten.
Ohne die Unterstützung von Seiten der pharmazeutischen Industrie wäre es nicht möglich, ein solches Register auf die Beine zu stellen, denn die Sozialversicherung unterstützt derartige Projekte in Österreich ebenso wenig wie in vielen anderen europäischen Ländern.

Die Daten aus BioReg wurden 2021 aktuell ausgewertet - was lässt sich daran ablesen?

LEEB: Zunächst zeigt sich, dass die Akzeptanz von BioReg unter den österreichischen Rheumatologen weiter zugenommen hat. Die COVID-19-Krise hatte keinen Einfluss auf Eingabehäufigkeit oder Eingabedisziplin. Im letzten Jahr wurden durchschnittlich vier Kontrolluntersuchungen von BioRegPatientinnen und -Patienten proTag durchgeführt und dokumentiert, darüber hinaus wurde praktisch an jedem Arbeitstag ein neuer Patient in BioReg eingeschlossen.

\) Die Akzeptanz von BioReg unter den österreichischen Rheumatologen hat weiter zugenommen

Tagesaktuell (28.04.2021) sind, wie auf der BioReg-Homepage (https:// www.bioreg.at/stats/) einzusehen, 3351 Patienten in BioReg dokumentiert, davon 1698 Patienten mit rheumatoider Arthritis, $710 \mathrm{~Pa}-$ tienten mit Psoriasis-Arthritis, 773 Patienten mit Spondylitis ankylosans und 170 Patienten mit "sonstigen“ entzündlichen rheumatischen Erkrankungen. 
Des Weiteren ist zu sehen, dass es den Patienten unter der Behandlung mit Biologika, Biosimilars und tsDMARDs in Österreich Gottlob gut geht. Sie alle befinden sich im Durchschnitt in einer niedrigen Krankheitsaktivität, unabhängig von ihrer Grunderkrankung. Auch das ist auf der BioReg-Homepage tagesaktuell anhand der aktuellen Durchschnittswerte der Krankheitsaktivitätsindices ablesbar.

\) 2020 wurde praktisch an jedem Arbeitstag ein neuer Patient in BioReg eingeschlossen

Erfreulicherweise kann man auch sagen, dass die Therapien generell gut verträglich sind. Wir haben für die Patienten mit rheumatoider Arthritis eine Nebenwirkungshäufigkeit von $24 \mathrm{AEs}$ (Adverse events) pro 100 Patientenjahren und 13 schwerwiegenden Nebenwirkungen pro 100 Patientenjahren, insgesamt gibt es auch keine neuen Sicherheitssignale im Register während der bis jetzt mitunter bereits elfährigen Beobachtungszeit.

Gibt es Unterschiede in der rheumatologischen Versorgung zwischen Österreich und Deutschland bzw. dem Rest Europas? Was läuft bei uns besonders gut und was weniger?

LEEB: Hier gibt es einen Zusammenhang mit den Medikamentenpreisen. Österreich ist ein Niedrigpreisland. In Österreich war daher die Versorgung mit Biologika, Biosimilars und tsDMARDs seit jeher gut, auch weil wir in der Verschreibung relativ frei sind.

In Deutschland etwa werden gemäß der S2e-Leitlinie auch Kombinationen konventioneller Basistherapeutika eingesetzt, bevor Biologika, Biosimilars oder tsDMARDs zur Anwendung kommen; erst nach unzureichendem Ansprechen zweier csDMARD-Therapien soll eine bDMARD- oder tsDMARD-The- rapie zum Einsatz kommen. Daher kommen Biologika, im Vergleich zu Deutschland, in Österreich etwa drei Mal so häufig zum Einsatz. Das ist auch ein Grund dafür, dass Biosimilars in Deutschland sehr wohl zu einer Veränderung der Therapiestrategien und wahrscheinlich auch zu einem früheren Zugang der Patienten zu bDMARDs geführt haben, während das in Österreich eigentlich nicht der Fall sein konnte, da schon vor Markteinführung der Biosimilars alle Patienten, die ein bDMARD brauchten, auch eines erhalten konnten. Das ist ein großer Unterschied.

In Österreich schließen wir Patienten zudem viel früher in Biologika-Therapien ein als es etwa in Deutschland oder Großbritannien möglich ist, nämlich bereits ab einer moderaten Krankheitsaktivität. Wir sind natürlich aufgerufen, diesen guten Zustand verantwortungsvoll zu handhaben, um ihn auch erhalten zu können.

Ein weiterer Unterschied zwischen Österreich und Großbritannien ist zum Beispiel, dass dort Patienten gar nicht zu einem Biologikum kommen, wenn sie nicht im britischen Biologika-Register aufgenommen sind. Das galt lange Zeit auch für Skandinavien und andere europäische Staaten, sodass die dortigen Register entsprechend mehr Daten beinhalten.

Werden die Patienten in Österreich überall gleich gut versorgt?

LEEB: Das lässt sich aus den Registerdaten nicht direkt ablesen, denn im BioReg ist nicht vermerkt, woher die Patienten kommen Für BioReg ist der Patient völlig anonymisiert, nur der behandelnde Arzt kennt die Identität der Patienten. BioReg wiederum kennt nur die Identität des behandelnden Arztes.

Man könnte nur aufgrund der Lokalisation des Zentrums eventuell einen Unterschied in der rheumatologischen Versorgung ausfindig machen. Nach den derzeitigen Analysen sind keine derartigen Unterschiede ersichtlich, aber natürlich gibt es eine unterschiedliche Dichte von Rheumatologen in Österreich. So finden sich im Großraum Wien sicher mehr Rheumatologen als in anderen Regionen Österreichs. In vielen Gegenden müssen Patienten größere Strecken zurückzulegen, um zu einem Rheumatologen zu kommen. Aber beim Rheumatologen gibt es, soviel lässt sich aus den BioReg-Daten herauslesen, dann keinen Unterschied in der Versorgung.

Sie sind Obmann des Vereins BioReg und von Anfang an dabei - was hat Sie bei den Datenanalysen der letzten Jahre am meisten überrascht?

LEEB: Es hätte mich negativ überrascht, wenn es in Österreich anders gelaufen wäre als global. Wir haben hierzulande weder besondere Sicherheitsprobleme noch besondere Unterschiede was die Effizienz betrifft.

Ein bisschen überraschend ist auch, dass das Verweilen auf den einzelnen Therapien relativ ähnlich ist auch zwischen konventionellen Basistherapeutika und Biologika, Biosimilars und tsDMARDs mit der kleinen Einschränkung, dass mit der Zeit das steigende Angebotes an Präparaten zu einer höheren Wechselbereitschaft führt.

》) Biologika kommen in Österreich etwa drei Mal so häufig zum Einsatz wie in Deutschland

Ein Ergebnis allerdings ist bemerkenswert:Kortisontherapien werden eigentlich nie aufgrund von Unwirksamkeit abgesetzt. Sie werden abgesetzt, weil sie wegen der verminderten Krankheitsaktivität nicht mehr notwendig sind oder aufgrund von Unverträglichkeit; aber bei der 
rheumatoiden Arthritis wirkt die Kortisontherapie in nahezu $100 \%$ der Fälle.

Was sind die derzeitigen TherapieEmpfehlungen im Zusammenhang mit COVID-19?

LEEB: Wir haben zum gegenwärtigen Zeitpunkt 37 COVID-19-Erkrankungen bei Registerpatienten dokumentiert und, Gott sei Dank, keine Todesfälle. Die Häufigkeit entspricht etwa 1,1\% aller dokumentierten Patienten und das ist deutlich weniger als in der Gesamtbevölkerung. Allerdings glaube ich, dass UnderReporting eher für diese Zahl verantwortlich ist als eine Protektion durch entzündliche rheumatische Erkrankungen. Von der Altersstruktur her sind die Patienten im mittleren Lebensalter, um die 50 Jahre, zwischen 25 und 86 Jahre alt.

\) Kortisontherapien

werden eigentlich nie aufgrund von Unwirksamkeit abgesetzt

Die Patienten erhalten unterschiedliche Präparate mit einem Überhang an TNF-Blockern, aber da die Mehrzahl der Patienten in BioReg mit TNF-Blockern behandelt wird, ist das nicht überraschend. Tatsächlich vermutet man bei TNF-Blockern zumindest eine gewisse protektive Funktion was den Verlauf der Erkrankung betrifft. Der älteste Patient im Register ist ein Patient aus meiner Ordination, der sich - bereits an COVID-19 erkrankt unwissenderweise seinen TNF-Blocker gespritzt hat. Der Patient hat berichtet, dass der TNF-Blocker für inn auch in Bezug auf die Virusinfektion hilfreich gewesen sei.

Für Patienten auf Biologika, Januskinase-Inhibitoren und Biosimilars besteht eigentlich kein Grund dieTherapie in irgendeiner Weise zu unterbrechen - außer bei der manifesten Infektion mit SARS-CoV-2, aber das gilt für alle Infektionen. Eine Ausnahme ist Rituximab. Mit dem B-Zell-Depletor Rituximab sind wir sehr zurückhaltend und versuchen Therapien aufzuschieben, weil für die Rituximab-Therapie doch manchmal negative Auswirkungen auf eine etwaige COVID-Infektion beschrieben wurden.

》) Die Dateneingabe

ins BioReg ist einfach, zeitsparend und User-freundlich

Was die Kortisontherapie betrifft, so würde ich glauben, dass sie in niedriger Dosis eher günstig ist für Patienten mit einer SARSCoV-2-Infektion. Kortison wird ja auch, in Form von Dexamethason oder demnächst vermutlich in Form von inhalativem Budesonid, therapeutisch eingesetzt.

Wenn jemand Daten in BioReg eingeben möchte - was ist zu tun?

LEEB: Wer BioReg-Mitglied werden möchte, findet auf der Homepage bioreg.at in der Rubrik "Ärzte“ den Antrag auf Aufnahme in BioReg. Unter der von BioReg zur Verfügung gestellten Nummer können dann Patienten sofort dokumentiert werden.

Die Dateneingabe ins BioReg ist einfach, zeitsparend und Userfreundlich. Die entsprechenden
Work Sheets sind auf der Home Page abrufbar und werden elektronisch übertragen. Die Dateneingabe umfasst vier Seiten pro Patient, die nach der Eingabe einfach gespeichert und damit sofort in der Datenbank abgelegt werden.

Vorteilhefterweise entspricht die Dokumentation in BioReg den Anforderungen, welche die Sozialversicherung an die Dokumentationspflicht der verschreibenden Ärzte stellt. Man kann das BioRegWork Sheet also auch lokal abspeichern und hat damit mit Sicherheit auch gleich ausreichend dokumentiert, warum ein Patient eine bestimmte Therapie erhält.

Das Gespräch führte

Mag. Tanja Fabsits

Das Interview gibt es auch zu hören - im „Hörgang”, dem Podcast von SpringerMedizin.at, unter anderem unter https://bit.ly/ 2RE05hi oder über iTunes, Spotify und Podigee.

\section{Weitere Informationen:}

www.bioreg.at

Hinweis des Verlags. Der Verlag bleibt in Hinblick auf geografische Zuordnungen und Gebietsbezeichnungen in veröffentlichten Karten und Institutsadressen neutral.

rheuma plus $2021 \cdot 20: 104-106$ https://doi.org/10.1007/s12688021-00428-9

(c) Springer-Verlag GmbH Austria, ein Teil von Springer Nature 2021 